\title{
Muscular involvement assessed by MRI correlates to motor function measurement values in oculopharyngeal muscular dystrophy
}

\author{
Arne Fischmann • Monika Gloor · Susanne Fasler • Tanja Haas • \\ Rachele Rodoni Wetzel · Oliver Bieri · Stephan Wetzel · Karl Heinimann • \\ Klaus Scheffler · Dirk Fischer
}

Received: 26 October 2010/Revised: 13 January 2011/Accepted: 25 January 2011/Published online: 22 February 2011

(c) Springer-Verlag 2011

\begin{abstract}
Oculopharyngeal muscular dystrophy (OPMD) is a progressive skeletal muscle dystrophy characterized by ptosis, dysphagia, and upper and lower extremity weakness. We examined eight genetically confirmed OPMD patients to detect a MRI pattern and correlate muscle involvement, with validated clinical evaluation methods. Physical assessment was performed using the Motor Function Measurement (MFM) scale. We imaged the lower extremities on a $1.5 \mathrm{~T}$ scanner. Fatty replacement was graded on a 4-point visual scale. We found prominent affection of the adductor and hamstring muscles in the thigh, and soleus and gastrocnemius muscles in the lower leg. The MFM assessment showed relative mild clinical impairment, mostly affecting standing and transfers, while
\end{abstract}

Arne Fischmann and Monika Gloor authors are contributed equally to this work.

Electronic supplementary material The online version of this article (doi:10.1007/s00415-011-5937-9) contains supplementary material, which is available to authorized users.

A. Fischmann $(\bowtie)$

Department of Neuroradiology, Institute of Radiology,

University of Basel Hospital, Petersgraben 4, 4031 Basel,

Switzerland

e-mail: fischmanna@uhbs.ch

M. Gloor · S. Fasler · T. Haas · O. Bieri · K. Scheffler Department of Radiological Physics, Institute of Radiology,

University of Basel Hospital, Basel, Switzerland

S. Wetzel

Department of Neuroradiology, Swiss Neuro Institute,

Hirslanden Klinik Zurich, Zurich, Switzerland

K. Heinimann

Department of Medical Genetics, University of Basel Children's

Hospital, Basel, Switzerland distal motor capacity was hardly affected. We observed a high (negative) correlation between the validated clinical scores and our visual imaging scores suggesting that quantitative and more objective muscle MRI might serve as outcome measure for clinical trials in muscular dystrophies.

Keywords MRI - Motor function measurement . Outcome measure $\cdot$ Muscle $\cdot$ Oculopharyngeal muscular dystrophy $\cdot$ OPMD

\section{Introduction}

Oculopharyngeal muscular dystrophy (OPMD) is a rare, slowly progressive autosomal dominant muscular dystrophy, although cases of autosomal recessive inheritance are reported [1]. Compared to Duchenne or limb girdle muscular dystrophies (LGMD), disease onset is late adult age with a mean age of 45 years (range 30-60 years), and disease

D. Fischer

Department of Neuropaediatrics, University Children's Hospital,

Basel, Switzerland

D. Fischer

Department of Neurology, University of Basel Hospital,

Basel, Switzerland

R. Rodoni Wetzel

Physiotherapy Neurology, University of Basel Hospital,

Basel, Switzerland 
progression in OPMD is relatively slow. It has an estimated prevalence of 1 in 100,000 worldwide [2], but it is much more common (1:1,000-1:600) in certain ethnic communities, such as French Canadians [3] and Bukhara Jews [4].

OPMD is caused by a mutation in the ubiquitously expressed Polyadenlyate binding protein-1 (PABPN1)-gene [5]. The clinical phenotype in OPMD, however, is restricted to skeletal muscles, especially to the cricopharyngeal and levator palpebrae muscles. The initial symptom in most cases is a slowly progressing ptosis, while later dysphagia due to muscular weakness might compromise oral food intake. Facial weakness and involvement of proximal lower extremity muscles are described in more advanced stages [6].

Muscular biopsies of OPMD patients showed rimmed vacuoles and intranuclear inclusions as well as atrophic and hypertrophic fibers. Predominance of Type 1 fibers is also common [7]. Diagnosis is usually made clinically and three criteria are required for a clinical diagnosis of autosomal dominant OPMD: a positive family history, the presence of ptosis, and the presence of dysphagia [8]. Definitive diagnosis depends upon genetic testing for a polyalanin-repeat expansion in the PABPN1-gene and the detection of more than 10 "GCN" trinucleotide repeats in the first exon of PABPN1 [9]. The triplet repeat in PABPN1 is stable, and triplet repeat expansion is uncommon. In contrast to many other triplet disorders, there is no known clinical anticipation in OPMD.

Recent studies on muscle imaging showed the usefulness of pattern recognition for the differential diagnosis of muscular dystrophies [10, 11]. Previous studies in OPMD describe proximal weakness and pelvic girdle involvement $[4,9]$. CT and MRI demonstrated fatty replacement of the shoulder girdle muscles $[12,13]$. However, thigh and lower leg muscle involvement has neither been studied systematically, nor compared to clinically validated tests or to the pattern of involvement in other hereditary myopathies.

The aim of this study was, therefore, to establish a pattern of muscular involvement in OPMD and to correlate fatty infiltration to validated clinical tests.

\section{Materials and methods}

\section{Patients}

Patients with OPMD that had been identified in the Department of Medical Genetics of the University of Basel Children's Hospital were invited to participate in this study. Eight genetically confirmed OPMD patients agreed and were recruited (age range 41-76 years, 6 female, 2 male). The youngest patient presented without symptoms of OPMD. Written informed consent was obtained from all patients. All measurements were approved by the local ethics committee (EKBB-Nr. 83/08).
Clinical assessment of muscle weakness

Physical assessment was performed prior to imaging in all but two patients, who did not wish to participate in this part of the study. We used the Motor Function Measurement (MFM) scale, which covers the whole range of abilities in both ambulant and non-ambulant patients. This scale comprised items from the following three dimensions: standing position and transfers, axial and proximal motor function, distal motor function. Each dimension is evaluated as a subset score, as well as a total score given in percentage points. It has been shown that this scale has a high inter-rater reliability, does not require any special equipment and is well tolerated by patients [14].

\section{Magnetic resonance imaging}

All imaging was performed on a $1.5 \mathrm{~T}$ whole body scanner (Magnetom Avanto, Siemens, Erlangen, Germany) with a dedicated 16-element matrix coil. Patients were placed with the knee joint in the center of the magnet. To permit reproduction for future follow-up examinations, two axial slice groups were placed centered at $14.5 \mathrm{~cm}$ below and $17.5 \mathrm{~cm}$ above the knee joint respectively, to include the largest diameter of the thigh and the lower leg. An imaging matrix of $384 \times 384$ and a field of view of $400 \times 400 \mathrm{~mm}^{2}$ were used, resulting in a $1 \mathrm{~mm}$ inplane resolution with $3 \mathrm{~mm}$ slice thickness. A T1 weighted turbo spin echo (TSE) sequence (TR $457 \mathrm{~ms}$, TE $7.5 \mathrm{~ms}$, Turbo factor 5, acquisition time 3:02 min) as well as a T2 weighted multi-contrast SE sequence (TR 1,330 ms, TE 10-320 ms, flip angle $=25^{\circ}$, acquisition time 2:23 $\mathrm{min}$ ) were acquired, and fat- and water-selective images were calculated to distinguish fatty infiltration in T2 from edematous changes.

Image evaluation

Images were digitally displayed on a diagnostic radiologic workstation with high-resolution monitors. All images were evaluated by one radiologist with 4 years of postfellow experience in neuromuscular imaging, who was blinded to clinical findings. Images were repeatedly evaluated after at least 1 month to assess intra-reader variability. Fatty replacement of muscle was graded on a 4-point scale derived from a scale proposed by Mercuri et al. [15] $(0=$ no fatty replacement; $1=$ minimal fatty replacement; $2=$ moderate fatty replacement less than $50 \%$ of muscle involvement; 3 = extensive fatty replacement of the muscle, more than $50 \%$ of muscle involved).

All muscles visible in the field of view were included in the evaluation, these comprised the thigh: quadriceps femoris (medial, lateral, intermediate vastus and rectus femoris), adductor magnus and longus, long and short head 
of the biceps femoris, semimembranosus and semitendinosus, gracilis and sartorius. In the lower leg we evaluated: soleus, medial and lateral part of the gastrocnemius, anterior and posterior tibial, long peroneal muscle. The flexor hallucis and digitorum longus as well as the extensor hallucis and digitorum longus, respectively were evaluated as one, because these muscles could not be reliably separated in MRI images. Each muscle was assigned a fatty replacement score, with separate scores for the left and right side.

In addition, the pattern of muscular involvement was compared to those of different myopathies established in the literature [10, 15-19].

\section{Statistical analysis}

Imaging scores were analyzed on a patient by patient basis due to symmetrical involvement of the legs. Arithmetic middle of the scores from the thigh, lower leg and whole leg were calculated and compared to clinical assessment of the MFM scale. Correlations of clinical and MRI scores were calculated as Pearson correlation coefficient with JMP 8.0.2 (SAS Institute, Cary (NC), USA).

\section{Results}

\section{Clinical data}

Eight patients with genetically proven OPMD were included into our study.

Seven patients were heterozygous carriers of a $(\mathrm{GCN})_{13}$ allele resulting in the insertion of 3 additional alanines into the PABPN1 protein. Patient 3 harboured a $(\mathrm{GCN})_{15}$ allele leading to an expansion of the polyalanine tract by 5 amino acids.

Mean age at onset of symptoms was 47 (40-50), average duration of symptoms was 20 years (10-35). The mean age at examination was 60 years, the median 62 years. Apart from patient 6 , who was a 41 year old presymptomatic patient, all other patients had symptoms and clinical weakness. All had a bilateral ptosis, different degrees of dysphagia and proximal leg weakness. Details on the clinical data are provided in Table 1. The total mean MFM score of all six examined patients was $94.0 \%$. The mean D1 (standing and transfers) subscore was $85.8 \%$; the D2 (axial and proximal motor capacity) subscore was $96.7 \%$; and the D3 (distal motor capacity) was 97.8\%. D1 was in all but one patient the lowest subscore, and D3 in all but another patient the highest subscore.

Muscle imaging findings

All but one patient (patient 6), presented with some degree of fatty muscular degeneration. Patient 6 only showed minimal fatty streaks bilaterally in the soleus. All affected patients displayed a similar pattern of muscular involvement with symmetric values in $98.5 \%-6$ of 400 muscles evaluated showed a 1 point difference (Table 2 and additional online material). At the thigh level fatty replacement was prominent in the adductor magnus and hamstring (semimembranosus, biceps femoris) muscles (Fig. 1). Patients with involvement of the vastus intermedius muscle showed fatty replacement mainly in the deeper parts of the muscle next to the femur (oblique arrows in Fig. 1). The gracilis (large arrow in Fig. 1) and the vastus medialis muscles were spared in all patients even in those with the most severe fatty infiltration. In the lower leg, the muscles of the posterior compartment were more involved than the muscles of the relatively spared anterior compartment. The most affected muscle was the soleus muscle followed by the medial gastrocnemius muscle, the lateral gastrocnemius, and peroneal muscles while the tibialis anterior muscle was relatively spared (Fig. 2). Similar to the pronounced intramuscular

Table 1 Clinical data of 8 patients with OPMD

\begin{tabular}{lllllllllll}
\hline Patient & Sex & $\begin{array}{l}\text { Age at } \\
\text { onset/years }\end{array}$ & $\begin{array}{l}\text { Age at } \\
\text { exam/years }\end{array}$ & Ptosis & Dysphagia & Weakness & MFM total & MFM D1 & MFM D2 & MFM D3 \\
\hline 1 & F & 40 & 75 & BP & CPM & - & 98 & 100 & 94 \\
2 & M & 45 & 69 & BP & ++ & +++ & 84 & 64 & 97 & 100 \\
3 & F & 49 & 59 & +++ & + & ++ & 97 & 92 & 100 & 97 \\
4 & F & 55 & 61 & BP & + & ++ & 92 & 77 & 92 & 100 \\
5 & F & 50 & 63 & BP & ++ & + & 93 & 82 & 97 & 90 \\
6 & M & - & 41 & - & - & - & 100 & 100 & 100 \\
7 & F & - & 54 & + & - & - & n.a. & n.a. & n.a. & n.a. \\
8 & F & 50 & 69 & BP & CPM & + & n.a. & n.a. & n.a. & n.a. \\
\hline
\end{tabular}

Patients 7 and 8 refused MFM measurements, n.a. = not applicable

MFM D1, MFM D2, MFM D3 motor function measurement with subscores in \%. CPM cricopharyngeal muscle; $B P$ bilateral ptosis; - no involvement, + mild involvement, ++ moderate involvement, +++ strong involvement 
Table 2 Degree of muscular fatty degeneration on a visual 4-point scale (0-3)

\begin{tabular}{|c|c|c|c|c|c|c|c|c|c|}
\hline Patient muscle & 1 & 2 & 3 & 4 & 5 & 6 & 7 & 8 & $\begin{array}{l}\text { Average muscular } \\
\text { fatty } \\
\text { degeneration }\end{array}$ \\
\hline Vastus lateralis & 0 & 1 & 0 & 0 & 0 & 0 & 1 & 0 & 0.25 \\
\hline Vastus intermedius & 0 & 2 & 0 & 0 & 0 & 0 & 1 & 0 & 0.375 \\
\hline Vastus medialis & 0 & 0 & 0 & 0 & 0 & 0 & 0 & 0 & 0 \\
\hline Rectus femoris & 0 & 1 & 0 & 0 & 0 & 0 & 0 & 0 & 0.125 \\
\hline Sartorius & 0 & 0 & 0 & 1 & 0 & 0 & 0 & 1 & 0.25 \\
\hline Adductor longus & 0 & 3 & 0 & 0 & 0 & 0 & 0 & 0 & 0.375 \\
\hline Adductor magnus & 1 & 3 & 2 & 2 & 2 & 0 & $2 *$ & 2 & 1.75 \\
\hline Gracilis & 0 & 0 & 0 & 0 & 0 & 0 & 0 & 0 & 0 \\
\hline Semimembranous & 1 & 3 & 0 & 1 & 1 & 0 & $2 *$ & 2 & 1.25 \\
\hline Semitendinous & 0 & 3 & 1 & 1 & 1 & 0 & 1 & 1 & 1 \\
\hline Long biceps & 0 & 3 & 1 & 1 & 1 & 0 & $1^{*}$ & 1 & 1 \\
\hline Short biceps & 0 & 2 & 0 & 1 & 0 & 0 & $1^{*}$ & 1 & 0.625 \\
\hline Anterior tibial & 0 & 0 & 0 & 0 & 0 & 0 & 0 & 0 & 0 \\
\hline Digital extensor & 0 & 1 & 0 & 0 & 0 & 0 & 0 & 0 & 0.125 \\
\hline Long peroneal & 0 & 2 & 0 & 0 & 1 & 0 & $1^{*}$ & 0 & 0.5 \\
\hline Posterior tibial & 0 & $0^{*}$ & 0 & 0 & 0 & 0 & 0 & 0 & 0 \\
\hline Digital flexor & 0 & 1 & 0 & 0 & 0 & 0 & 0 & 0 & 0.125 \\
\hline Soleus & 1 & 3 & 2 & 1 & 2 & 1 & 1 & 1 & 1.5 \\
\hline Medial gastrocnemius & 0 & 2 & 1 & 0 & 0 & 0 & 2 & 0 & 0.625 \\
\hline Lateral gastrocnemius & 0 & 1 & 0 & 0 & 0 & 0 & 1 & 0 & 0.25 \\
\hline Average muscular fatty degeneration & 0.14 & 1.45 & 0.32 & 0.36 & 0.36 & 0.045 & 0.64 & 0.41 & 0.47 \\
\hline Average thigh & 0.17 & 1.75 & 0.33 & 0.58 & 0.42 & 0 & 0.75 & 0.67 & 0.48 \\
\hline Average calf & 0.1 & 1.1 & 0.3 & 0.1 & 0.3 & 0.1 & 0.5 & 0.1 & 0.48 \\
\hline
\end{tabular}

* A small difference between right and left, usually with greater atrophy on the right side (see additional online material)

affection of the vastus intermedius muscle, the same characteristic variation was found in the gastrocnemius muscle, where deeper parts were also more prominently involved than the superficial parts. Details of muscle involvement scores of each patient and mean muscle involvement of each muscle are shown in Table 2. We found no edematous changes in any of the involved muscles.

\section{Correlation of clinical and MR findings}

The duration of symptoms weakly correlated to MRI scores $\left(R^{2}=0.3\right)$, while no correlation to MFM was detected. Especially, patient 1 with a long history since symptom onset had only minor changes in muscular MRI and in the MFM scores. As the mutation of this patient was identical to the other patients, there is currently no explanation for this phenomenon. However, fatty replacement and extent of muscle involvement increased with progressive peripheral weakness. Linear regression with Pearson's correlation coefficient between the MFM scores and the mean muscle affection score across all muscles as assessed by MRI is shown in Fig. 3. A strong negative correlation $R^{2}=-0.94$ was observed between the total MFM score and the imaging scores. The analysis of the subscores revealed that most of the correlation was based on the D1 (standing and transfers) with an $R^{2}$ of -0.87 while the D2 (axial and proximal motor capacity) subscore and the D3 (distal motor capacity) showed no correlation to muscular fatty degeneration with $R^{2}$ of -0.02 and 0.13 , respectively. Separate analysis of MRI for the thigh and lower leg and correlation to the MFM subscores revealed no additional negative correlation: the correlation of D1 with MRI involvement of the thigh muscles improved slightly to $R^{2}=-0.91$, while the correlation of D1 to the involvement of the lower leg showed a non significant $R^{2}=$ -0.77 . Omission of the patients 6 (who was asymptomatic) and 1 (who had a different clinical profile) reduced the level of confidence but had no major influence on correlations (additional online material).

\section{Discussion}

In this study we performed a systematic prospective muscle imaging assessment in eight patients with genetically proven OPMD. 


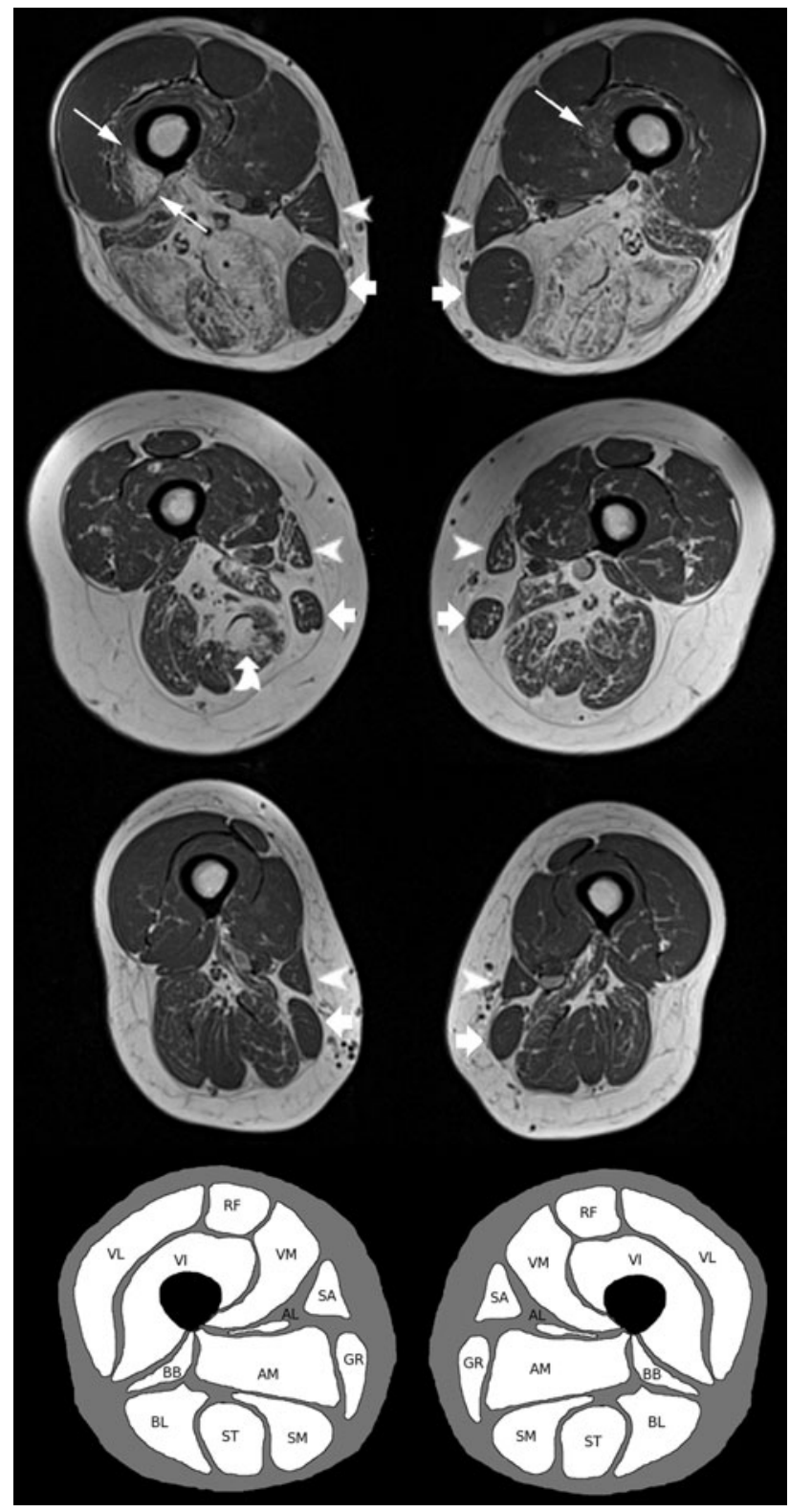

Fig. 1 T1 weighted images of the thigh with fatty replacement and atrophy in three patients showing different stages of involvement Bottom to top patient 3 (age 59 years, disease duration 10 years), patient 4 (age 61 years, disease duration 6 years), patient 2 (age 69 years, disease duration 24 years). Curved arrow lipoma in the right semimembranosus. $V L$ lateral vastus, $V I$ intermediate vastus, $V M$ medial vastus, $R F$ rectus femoris, $S A$ sartorius, $A L$ long adductor, $A M$ major adductor, $G R$ gracilis, $S M$ semimembranosus, $S T$ semitendinosus, $B L$ long biceps, $B B$ short

While in other myopathies with predominant proximal weakness (as LGMD) or myopathies with late adult onset (e.g. myofibrillar myopathies) a typical imaging pattern is described [18, 19], no such pattern is currently known for muscle involvement in OPMD. We could detect a characteristic pattern distinct from other known moyopathies as visualized in Figs. 1 and 2: At the thigh, we found early

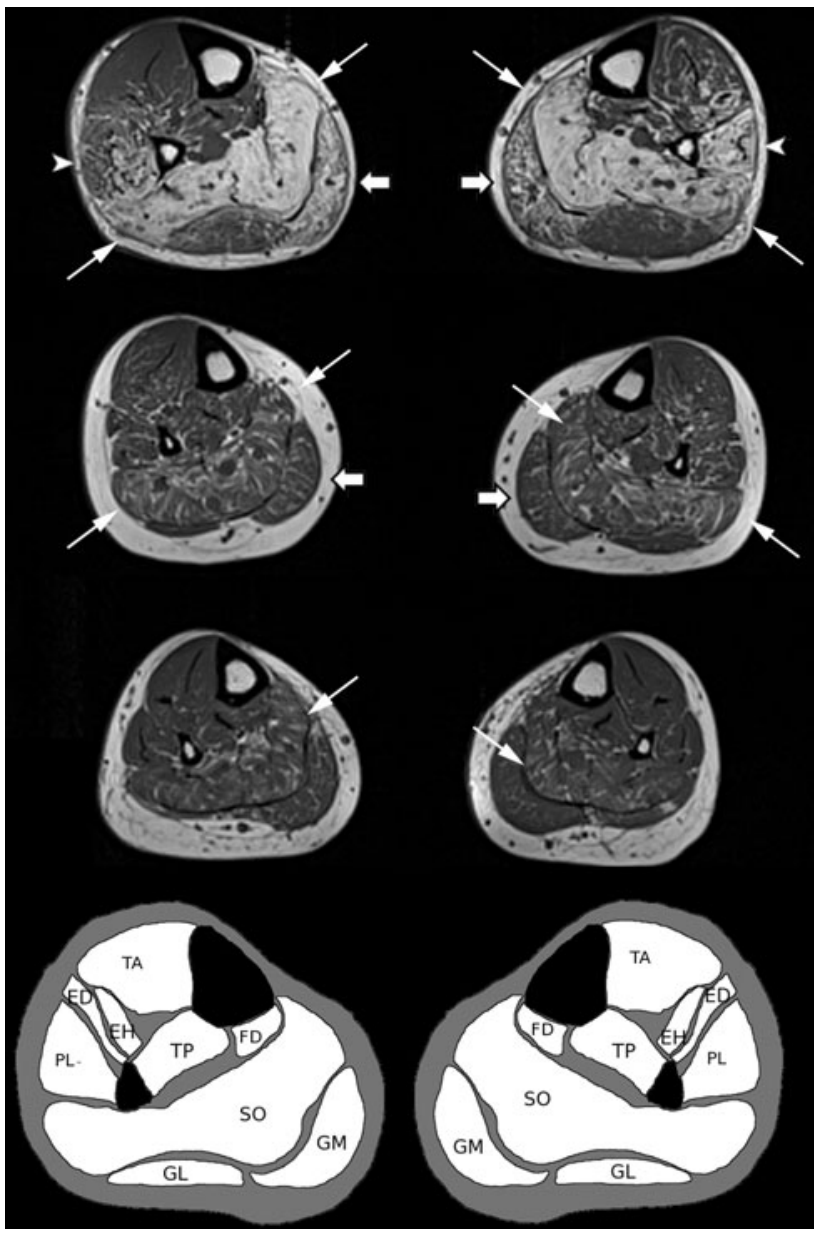

Fig. 2 Muscular MRI (T1 weighted sequences) of the lower leg of three patients with different stages of involvement from bottom to top patient 3 (age 59 years, disease duration 10 years), patient 4 (age 61 years, disease duration 6 years), patient 2 (age 69 years, disease duration 24 years), $T A$ anterior tibial, $T P$ posterior tibial, $E D$ extensor digitorum, $E H$ extensor hallucis, $P L$ long peroneal, $F D$ digital and hallux flexor, $S O$ soleus, $G M$ gastrocnemius (medial head), $G L$ gastrocnemius (lateral head)

and predominant involvement of the adductor magnus, semimembranosus, and biceps femoris muscles. In more severely affected patients also the semitendinosus muscle showed fatty infiltration, while sartorius and gracilis muscles were relatively spared in all patients. In the lower leg, the posterior compartment muscles showed more fatty infiltration than the anterior compartment muscles. The most affected muscle was the soleus muscle followed by the medial gastrocnemius muscle. In more severely affected patients also involvement of the lateral gastrocnemius and peroneal muscles was observed, while the tibialis anterior muscle was relatively spared. When comparing the pattern observed in OPMDs to other muscular dystrophies, predominant medial and posterior thigh and posterior lower leg involvement is also a typical finding in LGMD2A, LGMD2B, LGMD2I and Z-disc related myofibrillar 
Fig. 3 Correlation of MFMaggregate values and muscular fatty replacement on the visual scale show a high negative correlation. Left MFM total score by average muscular involvement. Right MFM D1 subscore by average muscular involvement of the thigh
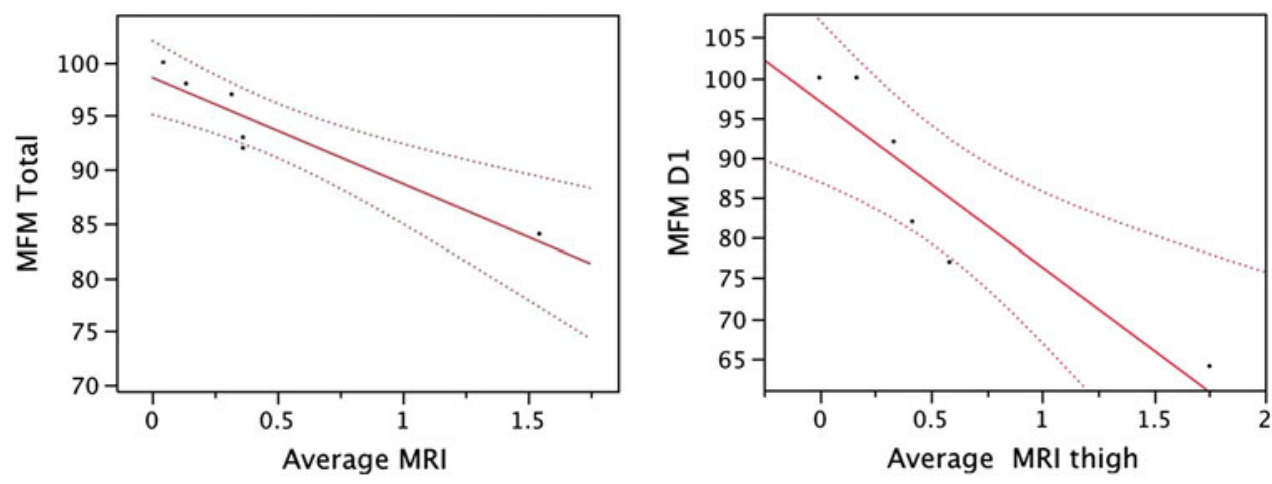

myopathies related to mutations in the myotilin, filamin-c, and ZASP genes. Distinction based on clinical findings between those entities usually is not difficult due to the pronounced ocular and bulbar affection in OPMD, the scapular winging often present in LGMD2A and LGMD2I, and the distal presentation most often found in Z-disc related myofibrillar myopathies. In addition to these clinical and pathologic findings subtle differences between the entities on muscle imaging are visible. In patients with involvement of the vastus intermedius and vastus lateralis muscles, we found predominant involvement of deep muscular layers, which has not been observed in myofibrillar myopathies [18], and in LGMD2I [18]. Nondystrophic myopathies on the other hand showed no muscular involvement despite severe clinical symptoms [20].

The most similar pattern to that observed in OPMD is the pattern described in LGMD2A [15, 18], where a similar involvement of the vastus intermedius muscle is found. Therefore, though the clinical presentation is absolutely different, a distinction between those two entities based solely on imaging findings of leg muscles seems hardly possible. Involvement of the posterior lower leg in LGMD2B was more prominent in the lateral gastrocnemius [17], in contrast to OPMD, where the medial head of the gastrocnemius was more affected than the lateral. In myotonic dystrophy (DM) type 1 the involvement of deep parts of the vastus intermedius as well as involvement of the posterior lower leg is similar to OPMD, while the hamstring muscles as well as the adductor magnus were rarely involved, which would facilitate the differentiation [16] and guide genetic testing, as the clinical presentation of myotonic dystrophies in early stages can sometimes resemble OPMD.

All patients in our trial presented with ptosis as initial symptom, and more variably with dysphagia and proximal leg muscle weakness. The mean age of onset at 47 in our study population was similar to other previous reports [8]; while, in contrast to our data, only half of the patients in France presented with ptosis [4]. Disease duration was variable (6-35 years) but did not correlate to the degree of peripheral muscle weakness. This finding is of interest as in OPMD, age of onset and progression are usually relatively constant [8]. The lack of correlation between onset and severity might also be caused by differences in the awareness of mild disabilities such as a beginning ptosis. To assess more objectively the clinical and functional impairment, we utilised the MFM, a validated and reliable clinical score, which covers a broad range of abilities in ambulant (and non-ambulant) patients. Using MFM we observed an overall relative mild mean affection. The subscores, however, showed the most involvement of standing and transfers, and to a lower degree affection of axial and proximal motor capacity, while motor abilities of distal muscles were hardly affected. This clinical pattern is in accordance to previous reports describing predominant proximal involvement in addition to the marked oculobulbar weakness [9]. The pattern of muscular weakness with predominant axial and proximal muscle involvement was also mirrored in our muscle MRI analysis.

To quantify the correlation of clinical and imaging data, fatty replacement scores were aggregated to a mean muscular involvement for the whole lower extremity and into subscores for the thigh and lower leg. The mean involvement across all muscles of an individual patient provides a broad impression of the degree of disease severity (Fig. 3). We could observe a high negative correlation of $R^{2}=$ -0.94 between the validated clinical scores and our visual imaging scores, indicating that an increased fatty degeneration of muscles correlates to reduced clinical abilities and impaired muscular function. While correlation of overall MFM and D1 subscores (transfer and axial functions) to imaging were strong, we found only a minimal correlation to the D2 and D3 subscores (proximal and distal motor functions). This might be due to the fact that the latter subcategories are evaluated with tasks involving mainly the upper extremities, and only the lower extremities were studied in MRI. As category D3 is rather dependent on fine motor skills than on overall muscle strength, it is unlikely that the correlation would have improved, if the upper extremities were to be included in the imaging evaluation. 
The strong correlation to clinical affection suggests that muscular MRI might serve as a surrogate outcome parameter for clinical involvement in trials of novel therapies which are currently under development. However, further studies including objective quantitative muscle MRI data are necessary to confirm and validate this observation. Potential quantitative methods as reference standards might include volumetric evaluation of fat percentage in musculature, T2 measurements in involved muscles or spectroscopic analysis of fat content. Future trials should preferably also include follow up cohorts to evaluate disease progress over time and correlate deteriorating clinical status to increased fatty infiltration on a single-patient basis.

In this context the findings in the presymptomatic patient 6 are especially interesting. While the genetic defect in this patient established the diagnosis, only minimal changes in the soleus muscle were visible. These changes might represent a precursor of future muscular involvement. Whether new imaging methods like diffusion weighted imaging or diffusion tensor imaging would improve presymptomatic detection remains to be established.

The major shortcoming of our study is the small number of patients stemming only from one region. In this context it would be advantageous to confirm the pattern of involvement in a different population.

Another limitation is based on the fact that only semiquantitative scores of muscular fatty replacement were used. This score is established for clinical evaluation of fatty replacement in neuromuscular disorders [15], but currently there are no published comparisons to different established scores for fatty replacement [21].

In conclusion, we found a consistent pattern of muscular involvement in the lower extremities in OPMD. Muscular involvement and fatty degeneration was most prominent in the proximal thigh muscles, with prominent affection of the adductor and hamstring muscles in the thigh, and less marked soleus and gastrocnemius muscle involvement in the lower leg. Furthermore, we observed a strong negative correlation between the validated clinical scores and our visual imaging scores suggesting that quantitative and more objective muscle MRI might serve as a potential biomarker and outcome measure for clinical trials in muscular dystrophies.

Acknowledgments The authors thank all patients for their willingness to participate in this study. We also would like to thank the reviewers for their helpful contributions that greatly improved the manuscript. D.F. is supported by a grant from the Lorenzo-PiaggioFoundation, Switzerland. M.G. is supported by the Swiss National Science Foundation, Grant 325230-118377.

Conflict of interest None.

\section{References}

1. Semmler A, Kress W, Vielhaber S, Schröder R, Kornblum C (2007) Variability of the recessive oculopharyngeal muscular dystrophy phenotype. Muscle Nerve 35(5):681-684

2. Fan X, Rouleau GA (2003) Progress in understanding the pathogenesis of oculopharyngeal muscular dystrophy. Can J Neurol Sci 30(1):8-14

3. Barbeau A (1969) Oculopharyngeal muscular dystrophy in French Canada (abstract of the presidential address). In: Brunette JR, Barbeau A (eds) Progress in neuro-ophthalmology Vol. 2. Excerpta Medica, Amsterdam, p 3

4. Fardeau M, Tomé FM (1997) Oculopharyngeal muscular dystrophy in France. Neuromuscul Disord 7:S30-S33

5. Brais B, Bouchard JP, Xie YG, Rochefort DL, Chrétien N, Tomé FM, Lafrenière RG, Rommens JM, Uyama E, Nohira O, Blumen S, Korczyn AD, Heutink P, Mathieu J, Duranceau A, Codère F, Fardeau M, Rouleau GA, Korcyn AD (1998) Short GCG expansions in the PABP2 gene cause oculopharyngeal muscular dystrophy. Nat Genet 18(2):164-167

6. Sieb JP, Schrank B (2009) Occulopharyngeal muscle dystrophy (in German). In: Sieb JP, Schrank B (eds) Neuromuscular disorders (in German). Kohlhammer, Stuttgart, pp 103-105

7. Tomé FM, Chateau D, Helbling-Leclerc A, Fardeau M (1997) Morphological changes in muscle fibers in oculopharyngeal muscular dystrophy. Neuromuscul Disord 7:S63-S69

8. Brais B, Xie Y, Sanson M, Morgan K, Weissenbach J, Korczyn AD, Blumen SC, Fardeau M, Tome FMS, Bouchard J, Rouleau GA (1995) The oculopharyngeal muscular dystrophy locus maps to the region of the cardiac alpha and beta myosin heavy chain genes on chromosome 14q11.2-q13. Hum Mol Genet 4(3):429

9. Brais B, Rouleau GA, Bouchard JP, Fardeau M, Tomé FM (1999) Oculopharyngeal muscular dystrophy. Semin Neurol 19(1):59-66

10. Mercuri E, Clements E, Offiah A, Pichiecchio A, Vasco G, Bianco F, Berardinelli A, Manzur A, Pane M, Messina S, Gualandi F, Ricci E, Rutherford M, Muntoni F (2010) Muscle magnetic resonance imaging involvement in muscular dystrophies with rigidity of the spine. Ann Neurol 67(2):201-208

11. Wattjes MP, Kley RA, Fischer D (2010) Neuromuscular imaging in inherited muscle diseases. Eur Radiol 20(10):2447-2460

12. King MK, Lee RR, Davis LE (2005) Magnetic resonance imaging and computed tomography of skeletal muscles in oculopharyngeal muscular dystrophy. J Clin Neuromuscul Dis 6(3):103-108

13. Bilgen C, Bilgen IG, Sener RN (2001) Oculopharyngeal muscular dystrophy: clinical and CT findings. Comput Med Imaging Graph 25(6):527-529

14. Bérard C, Payan C, Hodgkinson I, Fermanian J, MFMCS Group (2005) A motor function measure for neuromuscular diseases. Construction and validation study. Neuromuscul Disord 15(7):463-470

15. Mercuri E, Bushby K, Ricci E, Birchall D, Pane M, Kinali M, Allsop J, Nigro V, Sáenz A, Nascimbeni A, Fulizio L, Angelini C, Muntoni F (2005) Muscle MRI findings in patients with limb girdle muscular dystrophy with calpain 3 deficiency (LGMD2A) and early contractures. Neuromuscul Disord 15(2):164-171

16. Kornblum C, Lutterbey G, Bogdanow M, Kesper K, Schild H, Schröder R, Wattjes MP (2006) Distinct neuromuscular phenotypes in myotonic dystrophy types 1 and 2: a whole body highfield MRI study. J Neurol 253(6):753-761

17. Kesper K, Kornblum C, Reimann J, Lutterbey G, Schröder R, Wattjes MP (2009) Pattern of skeletal muscle involvement in primary dysferlinopathies: a whole-body 3.0-T magnetic resonance imaging study. Acta Neurol Scand 120(2):111-118

18. Fischer D, Kley RA, Strach K, Meyer C, Sommer T, Eger K, Rolfs A, Meyer W, Pou A, Pradas J, Heyer CM, Grossmann A, 
Huebner A, Kress W, Reimann J, Schröder RJ, Eymard B, Fardeau M, Udd B, Goldfarb L, Vorgerd M, Olivé M (2008) Distinct muscle imaging patterns in myofibrillar myopathies. Neurology 71(10):758-765

19. Fischer D, Walter MC, Kesper K, Petersen JA, Aurino S, Nigro V, Kubisch C, Meindl T, Lochmüller H, Wilhelm K, Urbach H, Schröder R (2005) Diagnostic value of muscle MRI in differentiating LGMD2I from other LGMDs. J Neurol 252(5):538-547
20. Kornblum C, Lutterbey GG, Czermin B, Reimann J, von KleistRetzow JC, Jurkat-Rott K, Wattjes MP (2010) Whole-body high-field MRI shows no skeletal muscle degeneration in young patients with recessive myotonia congenita. Acta Neurol Scand 121(2):131-135

21. Goutallier D, Postel JM, Bernageau J, Lavau L, Voisin MC (1994) Fatty muscle degeneration in cuff ruptures. Pre- and postoperative evaluation by CT scan. Clin Orthop Relat Res 304:78-83 